\title{
Measuring vorticity vector from the spinning of micro-sized mirror- encapsulated spherical particles in the flow
}

F SCI

Cite as: Rev. Sci. Instrum. 90, 115111 (2019); https://doi.org/10.1063/1.5121016

Submitted: 22 July 2019 . Accepted: 21 October 2019 . Published Online: 13 November 2019

Huixuan Wu, Haitao Xu, and Eberhard Bodenschatz

COLLECTIONS

F This paper was selected as Featured

SCI This paper was selected as Scilight
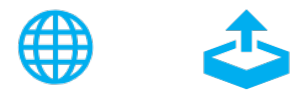

\section{ARTICLES YOU MAY BE INTERESTED IN}

Studying vorticity with mirror-embedded particles

Scilight 2019, 461105 (2019); https://doi.org/10.1063/10.0000275

A fully-adjustable picosecond resolution arbitrary timing generator based on multi-stage time interpolation

Review of Scientific Instruments 90, 114702 (2019); https://doi.org/10.1063/1.5119148

GaAs x-ray detectors with sub-nanosecond temporal response

Review of Scientific Instruments 90, 113505 (2019); https://doi.org/10.1063/1.5127294

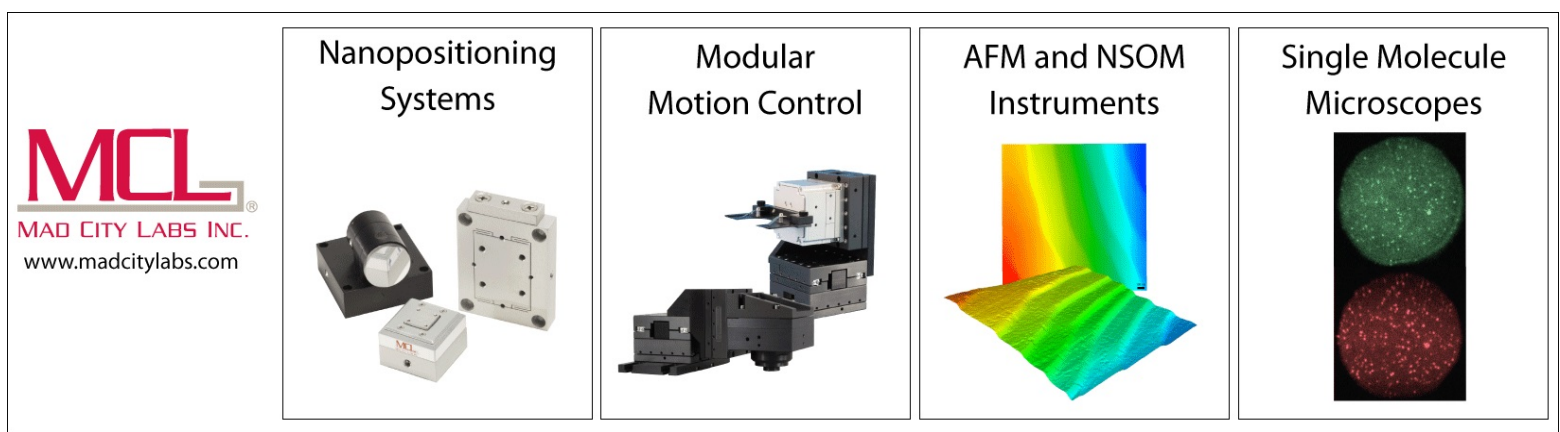




\title{
Measuring vorticity vector from the spinning of micro-sized mirror-encapsulated spherical particles in the flow
}

\author{
Cite as: Rev. Sci. Instrum. 90, 115111 (2019); doi: 10.1063/1.5121016 \\ Submitted: 22 July 2019 • Accepted: 21 October 2019 • \\ Published Online: 13 November 2019
}

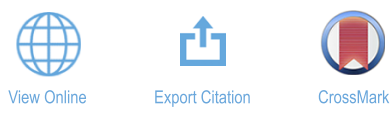

\author{
Huixuan Wu, ${ }^{1, \text { a) }}$ Haitao $X \mathbf{u}^{2,(b)}$ and Eberhard Bodenschatz ${ }^{3,4,5, c)}$

\section{AFFILIATIONS} \\ ${ }^{1}$ University of Kansas, Lawrence, Kansas 66049, USA \\ ${ }^{2}$ Center for Combustion Energy and School of Aerospace Engineering, Tsinghua University, Beijing 100084, China \\ ${ }^{3}$ Max Planck Institute for Dynamics and Self-Organization (MPIDS), 37077 Göttingen, Germany \\ ${ }^{4}$ Institute for Dynamics of Complex Systems, University of Göttingen, 37077 Göttingen, Germany \\ ${ }^{5}$ Laboratory of Atomic and Solid State Physics and Sibley School of Mechanical and Aerospace Engineering, \\ Cornell University, Ithaca, New York 14853, USA
}

\author{
a)hwu@ku.edu \\ b)hxu@tsinghua.edu.cn \\ c)eberhard.bodenschatz@ds.mpg.de
}

\begin{abstract}
We demonstrate a nonintrusive technique that is capable of measuring all three-components of vorticity following small tracer particles in the flow. The vorticity is measured by resolving the instantaneous spin of the microsized spherical hydrogel particles, in which small mirrors are encapsulated. The hydrogel particles have the same density and refractive index as the working fluid-water. The trajectory of the light reflected by the spinning mirror, recorded by a single camera, is sufficient to determine the 3D rotation of the hydrogel particle, and hence the vorticity vector of the flow at the position of the particle. Compared to more conventional methods that measure vorticity by resolving velocity gradients, this technique has much higher spatial resolution. We describe the principle of the measurement, the optical setup to eliminate the effect of particle translation, the calibration procedure, and the analysis of measurement uncertainty. We validate the technique by measurements in a Taylor-Couette flow. Our technique can be used to obtain the multipoint statistics of vorticity in turbulence.
\end{abstract}

Published under license by AIP Publishing. https://doi.org/10.1063/1.5121016

\section{INTRODUCTION}

The vorticity field underlies the dynamics of turbulent flows. Measuring vorticity experimentally, however, is notoriously difficult. ${ }^{1,2}$ Most available techniques obtain vorticity from fluid velocities at close distances. More than 60 years ago, Kavasznay started using four hot-wire probes to measure a vorticity component. ${ }^{3}$ With the advances of manufacturing technology, it is now possible to measure the full velocity gradient tensor using a miniature probe with an array of as many as 20 hot-wire sensors spanning over an approximately $5 \times 5 \mathrm{~mm}^{2}$ region. ${ }^{2,4}$ Overall, hot-wire based vorticity measurements provide a superior temporal resolution, but the method uses intrusive and delicate probes, which are severely limited in spatial resolution as they can hardly be made significantly smaller with current technologies.

Optical based velocimetry, such as laser Doppler velocimetry (LDV), has also been extended to measure the vorticity by utilizing multipoint measurements. ${ }^{5-7}$ Particle image velocimetry (PIV) ${ }^{8}$ and its variations, such as holographic PIV $^{9}$ and tomographic PIV, 8,10 are naturally suitable for measuring vorticity (or some of its components) through differentiation of the velocity fields. One interesting implementation of this method is the work reported by Zeff et al., ${ }^{11}$ who used three orthogonal laser sheets to construct three simultaneous planar PIV measurements at one location, from which they could access the whole velocity gradient tensor, which includes vorticity and rate-of-strain at a fixed location in a turbulent flow. 
All these methods measuring vorticity require that the velocity fields are well-resolved. A typical PIV setup usually has a spatial resolution of approximately $0.5-1 \mathrm{~mm}$. This resolution imposes a lower bound on the resolvable scale. For a given apparatus size, this translates to a limit on the Reynolds number. For example, the Taylor-microscale Reynolds number of the flow in the measurement of Zeff et al. ${ }^{11}$ is only 50 , for which the turbulent cascade is not well developed.

Vorticity can also be measured from the velocity-related signals at multiple points without actually resolving the velocities themselves. For example, when illuminated by an incident laser beam, two nearby particles with slightly different velocities induce different Doppler shifts to the scattered light, which then form a beating signal that can be used to deduce the velocity gradients. ${ }^{12,13}$ The autocorrelation of this interference signal, also called homodyne detection, therefore can be used to extract information about the probability distribution of the velocity gradients. ${ }^{14,15}$ When many particles are present in the measurement volume, the speckle pattern, formed by the interference of the scattered light and recorded by a fast camera, can be used to deduce the full instantaneous velocity gradient tensor. ${ }^{16}$ These methods, although they avoid explicitly measuring velocities at several points, still rely on the information provided by separate particles within a close distance. Therefore, they are also limited in spatial resolution.

An alternate way of measuring vorticity is to directly detect the spin of a single particle. ${ }^{17}$ Since vorticity equals twice the angular velocity of the local fluid element, measuring the rotation rate of a small spherical tracer is sufficient to obtain the vorticity. Recently, several groups used the light orbital angular momentum (OAM) to measure the angular velocity of a particle. ${ }^{18,19}$ The OAM of a laser slightly changes after reflection from a rotating object. Measuring this change provides the rotation rate. This technique requires the laser to be focused at a fixed point. The setup is similar to that of LDV. Therefore, it cannot follow a moving particle or provide the vorticity along a Lagrangian trajectory. In addition, direct imaging has also been used to measure the rotation of a sphere with surface markers ${ }^{20}$ and that of a symmetric nonspherical object (a jack), ${ }^{21}$ which give the vorticity sampled at the scale of the objects. These methods, however, are difficult to be used to measure true vorticity in high-Reynolds number turbulent flows as the objects must be large so that the surface marker or particle shape can be fully resolved on camera images. Another technique, magnetic particle tracking, is able to measure the rotation of a particle along its Lagrangian trajectory. ${ }^{22-24}$ However, the drawback is that the magnetic field decays as a cubic function of the distance from the magnet; hence, the source particle has to be large enough to be detectable in a laboratory scale experiment. Currently, only particles in the order of $1 \mathrm{~mm}$ are used. Their resolution and traceability are not enough.

A fourth method of spin detection, proposed by Frish and Webb, ${ }^{17}$ has the potential to provide Lagrangian vorticity information. To measure the rotation of the particle, they used thin mirrors encapsulated in transparent polymer beads that had the same reflective index as the working fluid. When illuminated by a laser beam, the reflection from the mirrors swept across two slots with known distance. By measuring the time lag between the light detected at the two slots, they could deduce one component of the rotation, and hence the related vorticity component. The system was able to measure one component of the vorticity at a fixed point. The advantage of this method was obviously the high spatial resolution determined by the particle diameter, which was easily orders of magnitude better than conventional indirect methods such as hot-wire arrays. The technique was later developed to measure all vorticity components using position sensitive photodiodes. ${ }^{25}$ The accuracy of photodiodes, however, was limited, and the method was left without further improvement.

Here, we present a major advance in pursuing the direct vorticity measurement technology. Using a novel optical setup, we can follow the reflection from the mirror-encapsulated particles while they move in the flow, in contrast with the previous fixed-point measurement. This development gives us access to the Lagrangian statistics of vorticity, which has long lacked experimental investigation. We show that, by recording the reflected light with state-of-theart high-speed digital cameras and analyzing with carefully designed algorithms, the accuracy of the vorticity measurement is well established. In Secs. II-IV, we describe the principle of the measurement, the optical setup to eliminate the effect of particle translation, the calibration procedure, and the analysis of measurement errors. We validate the technique by measurements in a Taylor-Couette flow.

\section{THE MIRROR-ENCAPSULATED PARTICLES AND THE MEASUREMENT PRINCIPLE}

\section{A. The particles}

The key to this vorticity measurement technique lies in the micro-mirror-capsules. According to the classical theory of Jeffery, only small spherical particles follow the flow rotation faithfully, i.e., being immune to the influence of strain. Hence, it is necessary to enclose the flat mirrors in transparent spherical capsules. The size of the particle should be smaller than the Kolmogorov length scale, which ranges roughly from 20 to $200 \mu \mathrm{m}$ in common turbulent flow experimental apparatus using water as the working fluid. We therefore developed a procedure to manufacture microparticles of diameters as small as $20 \mu \mathrm{m}$. In the validation experiments reported here, we used $72 \mu \mathrm{m}$ (on average) diameter particles. For a tracer particle, its response times should be smaller than the time scale of the local flow variation. Under the Stokes flow assumption, the particle translational relaxation time is $\tau_{u}=2 r^{2} \rho / 9 \mu$ and the rotational relaxation time is $\tau_{r}=2 r^{2} \rho / 15 \mu$, according to Brenner. ${ }^{27}$ Here, $r$ is the radius of the particle, $\rho$ is the density of the particle, and $\mu$ is the dynamic viscosity of the fluid. In water, our $72 \mu \mathrm{m}$ particle has translation and rotation relaxation times of $0.3 \mathrm{~ms}$ and $0.2 \mathrm{~ms}$, respectively. The typical Kolmogorov time scale, which also depends on the Reynolds number, is usually larger than $1 \mathrm{~ms}$. In addition, the particle ought to be transparent and neutrally buoyant. This requirement can be satisfied using polyacrylamide hydrogel material, which has a refractive index of 1.34 and a specific density of 1.05 .

We utilize the emulsion polymerization method to produce the hydrogel particle. Figure 1 shows one mirror-embedded particle under microscope. The particle shell is hardly visible due to the refractive index matching. The mean diameter is $72 \mu \mathrm{m}$, and the standard deviation of the diameter is $13 \mu \mathrm{m}$. Gravity sedimentation is used to narrow the distribution. The number of nonspherical or damaged particles is negligible. The diameter of the mirror disk is on average $30 \mu \mathrm{m}$, and their thickness is $1 \mu \mathrm{m}$. Although the mirror 


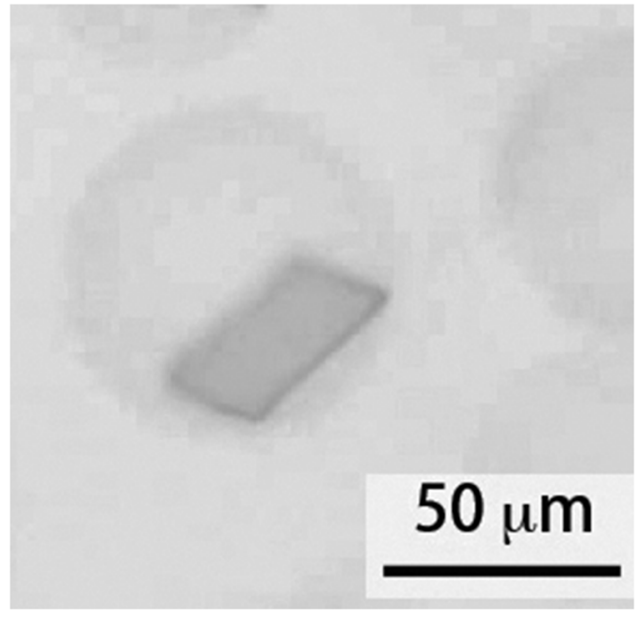

FIG. 1. A glass flake mirror embedded in a polyacrylamide hydrogel particle with a diameter of $70 \mu \mathrm{m}$. Due to refractive index matching, the hydrogel particle itself is hardly visible.

a

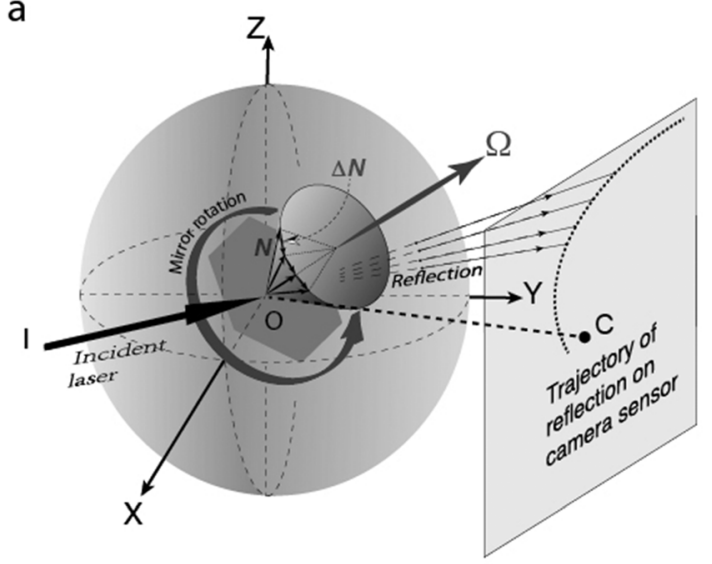

is enclosed inside the capsule, it may be off-center. This breaks the spherical symmetry, but the maximum perturbation to the momentum of inertia is actually less than $2 \%$, estimated with the dimension and density of the flake material.

Due to diffraction, the reflections of parallel beams from a finite size mirror diverge with a small angle, forming an airy pattern. This divergence angle depends on the relative size of the mirror, i.e., $\beta$ $\sim \lambda / D$, where $\lambda$ is the wavelength of the incident light and $D$ is the characteristic size of the mirror. For $\lambda=488 \mathrm{~nm}$ in our experiment, the estimated divergence angle is about $1^{\circ}$ for $D \approx 30 \mu \mathrm{m}$. This causes uncertainties in the determination of mirror orientation. The smaller the mirror, the larger the ambiguity. Diffractions from mirrors smaller than $20 \mu \mathrm{m}$ are found to be too large for proper analysis. This is the main reason that we set the minimum hydrogel particle size to $20 \mu \mathrm{m}$ in manufacturing.

\section{B. Measurement principle}

Figure 2(a) shows a sketch of the coordinate system. A mirror is located at the origin. It reflects the incident laser beam and leaves a light spot on a screen, which could be the sensor of a camera.

b

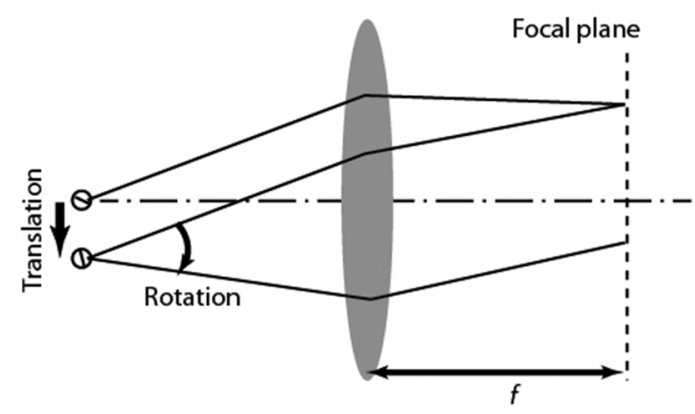

d

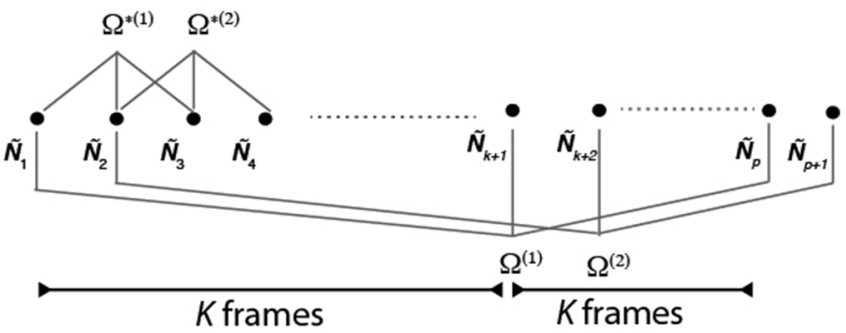

FIG. 2. Schematics of the optical setup. (a) The particle rotates around the axis $\Omega$; hence, the mirror norm vector $N$ forms a cone. The reflection marks a trajectory on the camera sensor. The incident laser is located in the $X-Y$ plane. The $X$ axis bisects the angle between the illumination and viewing direction. (b) Parallel beams are converged to a single point on the focal plane of a spherical convex lens; therefore, all translation information is filtered out and only rotation is recorded. (c) To determine the curvature center of an arc, any small perturbation may induce a large error. $\Delta \mathbf{N}$ s are the real increments of $\mathbf{N}$, and $\Delta \tilde{\mathbf{N}}$ s are the measured results. (d) $A$ strategy to improve accuracy is to calculate vorticity using the Ns over a large distance instead of using the neighboring ones. 
We define the viewing direction as the line OC, which connects the center of the mirror and the center of the screen. The viewing direction $\mathrm{OC}$ and the incident direction IO together define the X-Y plane, and the $\mathrm{X}$-axis is chosen to bisect the angle between IO and OC. The $\mathrm{Z}$-axis is determined by the right-hand rule. For simplicity, here, we consider rotation about the mirror center only. The effect of translation will be discussed later. As the mirror rotates with the angular velocity $\boldsymbol{\Omega}=\frac{1}{2} \boldsymbol{\omega}$, where $\boldsymbol{\omega}$ is the vorticity of the flow, its outward norm vector $\mathbf{N}$ forms a cone and the reflection spot leaves a trajectory on the screen. As the incident direction IO is fixed, there is a one-to-one correspondence between the reflected spot position on the screen and the direction of $\mathbf{N}$. Hence, measuring the trajectory of the reflected spot allows us to determine the rotation of $\mathbf{N}$, which consequently gives the angular velocity $\Omega$, and hence the vorticity $\boldsymbol{\omega}$.

To be specific, the tip of $\mathbf{N}$ forms a circle that is perpendicular to $\Omega$. As three points on the circle uniquely determine the circle, three successive measurements of Ns are theoretically enough to calculate $\boldsymbol{\Omega}$. Obviously, the variation of $\mathbf{N}$ over finite time, denoted as $\Delta \mathbf{N}$, is perpendicular to $\Omega$. This is evident from the kinetic equations $\dot{\mathbf{N}}=\boldsymbol{\Omega} \times \mathbf{N}$ so that $\dot{\mathbf{N}} \cdot \boldsymbol{\Omega}=(\boldsymbol{\Omega} \times \mathbf{N}) \cdot \boldsymbol{\Omega}=0$, where $\dot{\mathbf{N}}=d \mathbf{N} / d t$ is the rate of change of $\mathbf{N}$ with time. Hence, $\boldsymbol{\Omega}$ must be parallel to the direction of $\Delta \mathbf{N}_{1} \times \Delta \mathbf{N}_{2}$, where $\Delta \mathbf{N}_{1}=\mathbf{N}_{2}-\mathbf{N}_{1}$ and $\Delta \mathbf{N}_{2}=\mathbf{N}_{3}-\mathbf{N}_{2}$. The magnitude of $\boldsymbol{\Omega}$ can be determined by evaluating the effective rotation of $\mathbf{N}$ over a certain time. Given three successive measurements of $\mathbf{N}$, the angular velocity can, in principle, be calculated by

$$
\boldsymbol{\Omega}=\frac{1}{\Delta t} \arcsin \left(\frac{\left|\Delta \mathbf{N}_{1} \times \Delta \mathbf{N}_{2}\right|}{\left|\Delta \mathbf{N}_{1}\right|\left|\Delta \mathbf{N}_{2}\right|}\right) \frac{\Delta \mathbf{N}_{1} \times \Delta \mathbf{N}_{2}}{\left|\Delta \mathbf{N}_{1} \times \Delta \mathbf{N}_{2}\right|} .
$$

Here, $\Delta t$ is the time interval between successive images, and $|\boldsymbol{x}|$ indicates the magnitude of a vector $\boldsymbol{x}$. If $\Delta t$ is very small, Eq. (1) can be approximated by

$$
\boldsymbol{\Omega}=\frac{1}{\Delta t} \frac{\Delta \mathbf{N}_{1} \times \Delta \mathbf{N}_{2}}{\left|\Delta \mathbf{N}_{1}\right|\left|\Delta \mathbf{N}_{2}\right|},
$$

where we have used $\arcsin (x) \approx x$ for $x \rightarrow 0$.

Two subtleties in the above analysis need to be addressed. The first is the effect of particle translation, which would shift the light spot position on the screen even though the particle is not rotating, causing ambiguity in vorticity measurement. We eliminate this ambiguity by adding a spherical lens just one focal length in front of the camera sensor, as shown schematically in Fig. 2(b). This lens focuses all parallel beams to a single point, which eliminates all the translation-induced shift of the spot. Therefore, any movement of the spot must be due to the rotation of the mirror. Note that instead of the spherical lens, a concave spherical mirror can be used with the observation camera's sensor plane again in the focal plane (Fourier plane imaging). Indeed, concave mirrors are advantageous as they can cover larger solid angles. Thus, in the experimental system presented below, we used a concave mirror. The setup illustrated in Fig. 2(b) is insensitive to translation as long as the incident laser beam is collimated. The other subtlety is that the method described above would fail if the mirror norm is in the same direction of the vorticity vector. In this situation, $\mathbf{N}$ remains unchanged even though the particle is rotating. As a result, the spin cannot be detected. However, in real experiments, $\mathbf{N}$ is randomly distributed, having no correlation with $\boldsymbol{\Omega}$ at all. The probability of the situation that $\mathbf{N}$ and $\boldsymbol{\Omega}$ are experimentally indistinguishable is negligibly small.

\section{Analysis of the computational algorithm}

Our algorithm essentially reconstructs the circle formed by the tip of $\mathbf{N}$ based on the arc recorded by a finite size camera sensor. The primary uncertainty source is the error of $\mathbf{N}$ detection, as indicated in Fig. 2(c). If the arc is short, any small perturbation of $\mathbf{N}$ will jeopardize the curvature computation and the reliability of circle center positioning. Obviously, the longer arc is more robust. The following algebraic derivation clearly shows this relation. It also shows that the accuracies of two components [the $\mathrm{Y}$ and $\mathrm{Z}$ components in Fig. 2(a)] are much better than that of the third component $\mathrm{X}$.

A solid body rotation in three dimensions can be generated by the angular velocity $\boldsymbol{\Omega}$ or the skew-symmetric rotation matrix $\overline{\boldsymbol{\Omega}}$,

$$
\overline{\mathbf{\Omega}}=\left(\begin{array}{ccc}
0 & -\Omega_{3} & \Omega_{2} \\
\Omega_{3} & 0 & -\Omega_{1} \\
-\Omega_{2} & \Omega_{1} & 0
\end{array}\right),
$$

in which $\Omega_{i}$ s are the components of $\Omega$. Over a certain time $\tau$, mirror norm vector $\mathbf{N}_{0}$ is rotated to a new vector $\mathbf{N}_{1}=e^{\bar{\Omega} \tau} \mathbf{N}_{0}$, and then $\mathbf{N}_{2}=e^{2 \overline{\mathbf{\Omega}} \tau} \mathbf{N}_{0}$. Therefore, $\Delta \mathbf{N}_{2}=e^{\overline{\mathbf{\Omega}} \tau} \Delta \mathbf{N}_{1}$. The experimentally measured mirror norm $\tilde{\mathbf{N}}$ differs from the true normal vector $\mathbf{N}$, and the two vectors can be related, in general, by $\tilde{\mathbf{N}}=\mathbf{P N}$, with $\mathbf{P}$ being a matrix. As both $\mathbf{N}$ and $\tilde{\mathbf{N}}$ are unit vectors, it is obvious that for small uncertainties, i.e., $|\mathbf{N}-\tilde{\mathbf{N}}| \ll 1$, the matrix $\mathbf{P}$ can be expressed as follows to the first order:

$$
\mathbf{P} \approx \mathbf{I}+\varepsilon\left(\begin{array}{ccc}
0 & -p_{3} & p_{2} \\
p_{3} & 0 & -p_{1} \\
-p_{2} & p_{1} & 0
\end{array}\right)=\mathbf{I}+\varepsilon \mathbf{Q},
$$

where $\mathbf{I}$ is the unit matrix and $\varepsilon \ll 1$.

The quantity in the actual calculation of the angular velocity is the cross product of $\Delta \mathbf{N}_{1}$ and $\Delta \mathbf{N}_{2}$,

$$
\begin{aligned}
\Delta \tilde{\mathbf{N}}_{1} \times \Delta \tilde{\mathbf{N}}_{2} & =\left(\tilde{\mathbf{N}}_{1}-\tilde{\mathbf{N}}_{0}\right) \times\left(\tilde{\mathbf{N}}_{2}-\tilde{\mathbf{N}}_{1}\right) \\
& =\left(\mathbf{P}_{1} \mathbf{N}_{1}-\mathbf{P}_{0} \mathbf{N}_{0}\right) \times\left(\mathbf{P}_{2} \mathbf{N}_{2}-\mathbf{P}_{1} \mathbf{N}_{1}\right) \\
& =\left(\mathbf{P}_{1} \mathbf{N}_{1}-\mathbf{P}_{0} \mathbf{N}_{0}\right) \times\left(\mathbf{P}_{2} e^{\overline{\mathbf{\Omega} \tau}} \mathbf{N}_{1}-\mathbf{P}_{1} e^{\overline{\mathbf{\Omega} \tau}} \mathbf{N}_{0}\right) .
\end{aligned}
$$

Since both $\tau$ and $\varepsilon$ are much smaller than 1, the infinitesimal rotation operators, $\mathbf{P}$ and $e^{\bar{\Omega} \tau}$, commute to the accuracy of the first order, i.e., $\mathbf{P} e^{\overline{\mathbf{\Omega}} \tau} \approx e^{\overline{\mathbf{\Omega}} \tau} \mathbf{P}$. Therefore, keeping only the first order terms, the above equation turns out to be

$$
\begin{aligned}
\Delta \tilde{\mathbf{N}}_{1} \times \Delta \tilde{\mathbf{N}}_{2} \approx & \left(\mathbf{P}_{1} \mathbf{N}_{1}-\mathbf{P}_{0} \mathbf{N}_{0}\right) \times\left(e^{\overline{\mathbf{\Omega}} \tau} \mathbf{P}_{2} \mathbf{N}_{1}-e^{\overline{\mathbf{\Omega}} \tau} \mathbf{P}_{1} \mathbf{N}_{0}\right) \\
= & {\left[\left(\mathbf{N}_{1}-\mathbf{N}_{0}\right)+\varepsilon\left(\mathbf{Q}_{1} \mathbf{N}_{1}-\mathbf{Q}_{0} \mathbf{N}_{0}\right)\right] } \\
& \times\left[e^{\overline{\mathbf{\Omega}} \tau}\left(\mathbf{N}_{1}-\mathbf{N}_{0}\right)+e^{\overline{\mathbf{\Omega}} \tau} \varepsilon\left(\mathbf{Q}_{2} \mathbf{N}_{1}-\mathbf{Q}_{1} \mathbf{N}_{0}\right)\right] \\
= & \Delta \mathbf{N}_{1} \times \Delta \mathbf{N}_{2}+\varepsilon\left(\mathbf{Q}_{1} \mathbf{N}_{1}-\mathbf{Q}_{0} \mathbf{N}_{0}\right) \times\left[e^{\overline{\mathbf{\Omega}} \tau}\left(\mathbf{N}_{1}-\mathbf{N}_{0}\right)\right] \\
& \left.+\left(\mathbf{N}_{1}-\mathbf{N}_{0}\right) \times e^{\overline{\mathbf{\Omega}} \tau} \varepsilon\left(\mathbf{Q}_{2} \mathbf{N}_{1}-\mathbf{Q}_{1} \mathbf{N}_{0}\right)\right] \\
= & \Delta \mathbf{N}_{1} \times \Delta \mathbf{N}_{2}+\mathbf{V}
\end{aligned}
$$


in which $\mathbf{V}$ includes the first order terms of $\varepsilon$ or $\varepsilon \tau \Omega$. Careful evaluation of $\mathbf{V}$ shows that $V_{X} \sim \varepsilon|\Delta N|$, while $V_{Y}$ and $V_{Z} \sim \tau \Omega \varepsilon|\Delta N|$. Here, $\Omega$ is the magnitude of $\Omega$ and we assume that both $\left|\Delta \mathbf{N}_{1}\right|$ and $\left|\Delta \mathbf{N}_{2}\right|$ $\approx|\Delta N|$. It is clear that the $V_{Y}$ and $V_{Z}$ components are much smaller than $V_{X}$ since $\tau \Omega \ll 1$ in high speed sampling. The relative errors can be estimated from Eq. (2) as

$$
E_{X} \sim \frac{V_{X} /\left(|\Delta N|^{2} \tau\right)}{\Omega} \sim \frac{1}{\tau \Omega} \frac{\varepsilon}{|\Delta N|}
$$

and

$$
E_{Y}, E_{Z} \sim \frac{V_{Y, Z} /\left(|\Delta N|^{2} \tau\right)}{\Omega} \sim \frac{\varepsilon}{|\Delta N|}
$$

To obtain numerical values of these relative errors, we can estimate the uncertainty of the mirror norm vector to be $0.1^{\circ}$, which is about $10 \%$ of the diffraction-induced divergence angle. Therefore, $\varepsilon \approx 1.7 \times 10^{-3} \mathrm{rad}$. If we tune the recording speed high enough so that $\mathbf{N}$ travels $0.1^{\circ}$ per frame, then $\tau \Omega$ is also $0.1^{\circ}$ and $\Delta N \approx 1.7$ $\times 10^{-3} \mathrm{rad}$. Substituting these numbers to the above equation, we find that $E_{X}$ is on the order of 500, and $E_{Y}, E_{Z}$ are on the order of 1. These enormous errors indicate that using three successive measurements of $\mathbf{N}$ to calculate the vorticity is impractical. An effective way to improve the accuracy is to use the $\mathrm{Ns}$ from a longer arc. As shown in Fig. 2(d), we select three $\mathbf{N}$-vectors with $K$-frames spacing instead of using neighboring ones. The difference between the two vectors in this condition is $K \Delta \mathbf{N}$. As a result, the uncertainty is reduced by a factor of $K^{-1}$. Every such group of three Ns produces one angular velocity $\Omega^{(i)}$, and there are roughly $K$ groups along each trajectory. We then take the average of all these $\Omega^{(i)}$ s to yield the final $\Omega$. This further decreases the uncertainty by a factor of $K^{-\frac{1}{2}}$. The final uncertainty is reduced to the order of $K^{-\frac{3}{2}} \varepsilon /|\Delta N|=\varepsilon /\left(L K^{\frac{1}{2}}\right)$. Here, $L$ $=K|\Delta N|$ is measured in radians. For example, the uncertainty of vorticity determined from a $20^{\circ}$-arc trajectory, in which the mirror normal vector $N$ sweeps $20^{\circ}$ with increment $|\Delta N|=0.1^{\circ}$, is about $3 \%$ for $\mathrm{Y}$ and $\mathrm{Z}$ components and $15 \%$ for the $\mathrm{X}$ component. Nevertheless, the estimation above is based on the assumption that the position errors on spot centers are independent, which may not be true for centers determined from real images. Therefore, we would expect that the error should grow faster than $L^{-1} K^{-\frac{1}{2}}$ when $L$ and $K$ decrease. The empirical threshold value of $L K^{\frac{1}{2}}$ needs to be tuned by experiments. Note that the computation algorithm [Eq. (1)] does not require $\tau$ to be infinitesimal; this alternate method does not need high order term corrections.

One may notice that using an entire trajectory to calculate one $\omega$ requires the angular velocity to be constant throughout this trajectory. In the case that the vorticity varies during the time interval of recording one trajectory, the result $\omega$ will be a time-averaged value. If we denote the recorded angular range by $\Theta$ in radian, we note that $\Theta$ is usually smaller than 1 . The vorticity magnitude in homogeneous isotropic turbulence is in the order of $\left\langle\omega^{2}\right\rangle^{\frac{1}{2}} \approx 1 / \tau_{\eta}$, so the recording time interval is $\tau=\Theta /\left\langle\omega^{2}\right\rangle^{\frac{1}{2}}<\tau_{\eta}$, i.e., the interval of recording is shorter than the smallest time scale of the flow; hence, the vorticity hardly changes in this time. There is no appreciable loss of temporal resolution when reporting only one "mean" vorticity from a recorded trajectory.

\section{EXPERIMENTAL SETUP}

\section{A. Calibration}

To carry out experimental tests of this innovative technology, we have two more problems to solve. The first is how to deduce the mirror norm vector $\mathbf{N}$ from the reflection spot position recorded on an image. In other words, the question is to find a mapping from a pixel position to a unit vector $\mathbf{N}$. Our solution uses a mirror array, in which all the mirror's orientations are known [Fig. 3(c)]. When illuminated, the mirror array creates a column of spots on the screen. Consequently, we rotate the mirror array along the axis shown in Fig. 3(c). A light spot pattern can be generated [Fig. 3(d)]. The image is inverted, so the light spots are black. Every spot corresponds to a given normal vector $\mathbf{N}$. In between the light spots, the mapping can be obtained using a two dimensional cubic polynomial interpolation. Additional tests using a mirror on a rotary stage show that this mapping function introduces less than $1 \%$ uncertainty to the normal vector results.

The second problem is how to enlarge the angular field of view. As discussed before, a larger field (and a longer spot trajectory) can provide more accurate results. If the mirror rotates $1^{\circ}$, the reflection beam changes $2^{\circ}$. Hence, to enclose a $19^{\circ} \times 17^{\circ}$ solid angular range, the optical system has to cover approximately $40^{\circ} \times 35^{\circ}$, which cannot be achieved using a normal photography camera lens because of their small diameter. The solution is to use a spherical concave mirror as a light collector. As shown in Fig. 3(a), the measurement field is closer to the light collector than the camera. Therefore, a large angular field of view is compressed to a smaller one and fed into the camera. The diameter of our mirror is $156 \mathrm{~mm}$, its curvature is $1 / 325 \mathrm{~mm}^{-1}$, and its focal length is $162.5 \mathrm{~mm}$.

\section{B. Apparatus}

The Taylor-Couette flow in concentric cylinders has an analytical solution at low Reynolds numbers and displays a variety of transitions at higher Re. This is a good testbed for our vorticity measurement technique. The Taylor Couette apparatus and optical arrangement are shown in Fig. 3(a). The outer cylinder is stationary, and the inner cylinder rotates with a prescribed speed. The radius ratio is $\eta=r_{i} / r_{o}=0.58$. The Reynolds number defined by the inner cylinder speed is $R e=W r_{i}^{2} / v$. The inner cylinder radius $r_{i}=29.75 \mathrm{~mm}$, and $W$ is the angular velocity of the inner cylinder driven by an electric motor. A $75 \%$ glycerol-water solution is used as the working fluid in our test experiments. The fluid temperature is $22.1^{\circ} \mathrm{C}$. Its viscosity is $v=2.63 \times 10^{-3} \mathrm{~m}^{2} / \mathrm{s}$. At low Reynolds numbers, the analytical solution of the velocity profile is $v_{\theta}=A r+B / r$, where $A=r_{i}^{2} W /\left(r_{i}^{2}-r_{o}^{2}\right)$ and $B=r_{i}^{2} r_{o}^{2} W /\left(r_{o}^{2}-r_{i}^{2}\right)$. The other two components are $v_{z}=v_{r}=0$. The vorticity then is

$$
\begin{aligned}
\omega_{z}=\frac{\partial\left(r v_{\theta}\right)}{r \partial r}-\frac{\partial v_{r}}{r \partial \theta} & =2 A \\
\omega_{r} & =\omega_{\theta}=0 .
\end{aligned}
$$

Notice that $\omega_{z}, \omega_{\theta}$, and $\omega_{r}$ in the stable analytical solution do not vary over the axial or radial positions. Given the geometry of our device, we calculate the critical Reynolds number to be about 72 , according to the theoretical evaluation by Recktenwald. ${ }^{28}$ In our flow, this critical Reynolds number is slightly larger, at approximately 80 , which most likely is due to the effect of the side walls 
a

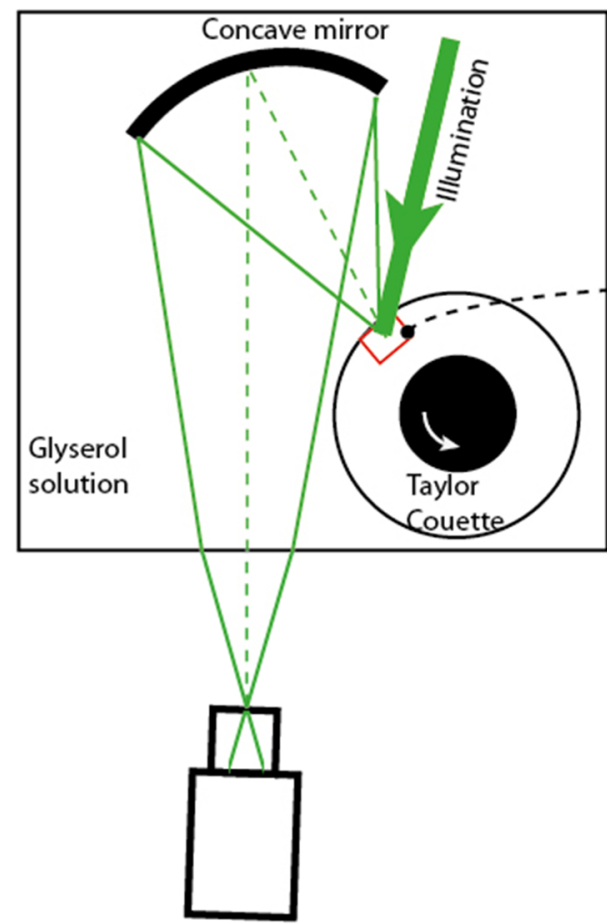

b

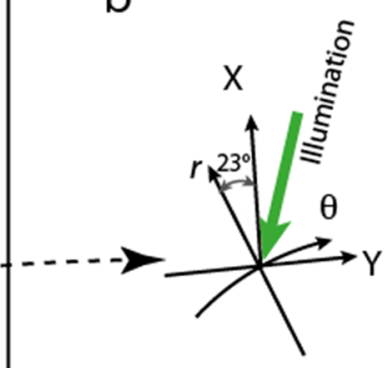

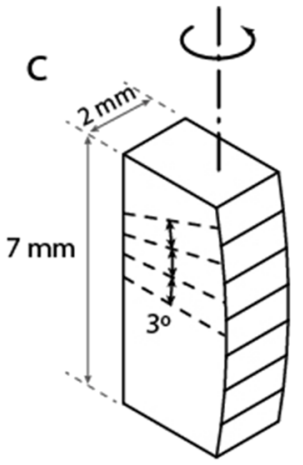

d

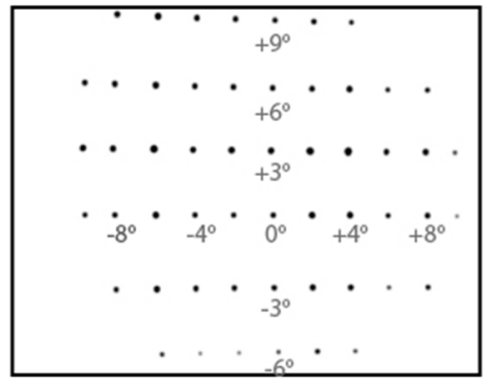

Camera

e

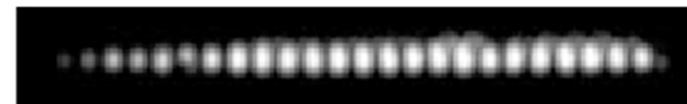

FIG. 3. The Taylor-Couette device and the validation experiment setup. (a) The arrangement of optics and camera. (b) Definition of coordinates. The vorticity is computed in the XYZ frame as described in Fig. 2(a). This frame is slightly different from the cylindrical frame determined by the Taylor Couette device. (c) The geometry of the calibration target. It is made of a mirror array aligned on a curve line. The angle between neighboring mirrors is $3^{\circ}$. To generate a two-dimensional reflection pattern, it is mounted on a motorized rotary stage. (d) The calibration image, each light spot corresponds to a known mirror norm direction. For clarity, we have enhanced and inverted the image. (e) One sample light spot trajectory captured by the high speed camera. It consists of 24 overlapped frames. The light spot sweeps $12^{\circ}$.

as the theory is for infinitely long devices. Above the critical value, Taylor vortices appear. At a higher Re number, Taylor vortices begin to show a wavy pattern. In our tests, the investigated flow is in the Couette and Taylor vortex regimes.

Figure 3(a) depicts the entire experimental setup. As previously mentioned, a concave mirror is used to collect the reflections from the tracers. This light collector is located near the Taylor-Couette device and far from the camera. It converges parallel beams to a single point on its focal plane, eliminating the effect of particle translation. The camera records the light-spot trajectory. The concave light collector and Taylor-Couette device are both submerged in the glycerol solution to minimize the light refraction due to the curved cylinder surface. The concave mirror is slightly rotated so that the light beam can reach the camera without being blocked by the outer cylinder. As a result, the coordinate system $(X, Y, Z$ frame) slightly differs from the cylindrical frame of the device $(r, \theta, z$ frame). Our $Z$ still coincides with the cylindrical axial direction $z$, so the measured vorticity $\omega_{Z}=\omega_{z}$, i.e., at low Re number, $\omega_{Z}=2 r_{i}^{2} W /\left(r_{i}^{2}-r_{o}^{2}\right)$. The $\mathrm{X}$ direction deviates from the radial direction by $23^{\circ}$, so $\omega_{X}=\omega_{r} \cos \left(23^{\circ}\right)+\omega_{\theta} \sin \left(23^{\circ}\right)$, and $\omega_{Y}=\omega_{\theta} \cos \left(23^{\circ}\right)-\omega_{r} \sin \left(23^{\circ}\right)$. At low Re number, they are both 0 . The camera is located outside the container. A $10 \times 10 \times 10 \mathrm{~mm}^{3}$ measurement volume is located near the outer cylinder and slightly above the midheight of the device. This domain is illuminated by a collimated laser beam.

The measurement volume is chosen to be close to the stationary outer cylinder where the flow velocity is small. and hence the tracer particles can stay in the measurement volume for relatively long times, enough for their rotation to be recorded on the camera sensor. For the Taylor-Couette flow at low Reynolds numbers, the vorticity is nearly a constant across the gap between cylinders. Therefore, the size of the measurement volume is not critical when evaluating the accuracy of the method. If the method is to be applied to more spatially in homogeneous flows, smaller measurement volume size should be used to reduce the error due to spatial variation of flow vorticity. In that case, care must be taken to ensure that the residence time of the tracer particle in the measurement volume is still sufficiently long for a reasonable number of mirror directions to be recorded by the optical system in order to calculate vorticity with a good accuracy.

The calibration of this setup has been described in Subsection III A. After calibration, we inject the microcapsules into the TaylorCouette cylinders. Figure $3(\mathrm{e})$ shows a sample light spot trajectory. 
It is an overlap of 24 frames recorded by a high speed camera. The mirror flakes may be noncircular, so the reflection spot is generally elliptical. The widely spread reflections from extremely small or strip-shape mirrors are filtered out by image processing as they cause large errors. Regarding the data processing, we define the location of the spot using the center of mass and fit the trajectory with a third-order polynomial to filter out high frequency noise.

\section{MEASUREMENT ACCURACY}

\section{A. Uncertainty}

It should be emphasized that optical vorticity probing is a general vorticity measurement technique. The Taylor-Couette flow serves as a testbed, but the error analysis in this section is valid for all conditions. We can obtain the uncertainty by comparing the measurement results at low $R e$ numbers to the analytical solution [Eq. (5)]. The relative error of the $i$ th component is defined as $E_{i}$ $=\left|\omega_{i}-\omega_{\text {Ana, } i}\right| /\left|\boldsymbol{\omega}_{A n a}\right|$. The numerator is the magnitude of the differences, and the denominator is the magnitude of the analytical solution $\left|\boldsymbol{\omega}_{\text {Ana }}\right|$. As aforementioned, the uncertainty should increase slightly faster than $L^{-1} K^{-1 / 2}$ as $L$ and $K$ decrease. Figure 4 confirms this result. The data used here are from the $R e=78$ case.

When the spot trajectory is extremely short, the error can be many times larger than the signal. However, it quickly decreases with a longer $L$ and larger $K$ until it reaches the minimum at $L K^{1 / 2}>$ 0.8 . Actually, the errors of $\mathrm{Y}$ and $\mathrm{Z}$ components reach an acceptable value of $5 \%-8 \%$ at $L K^{1 / 2}>0.4$. Data of other Reynolds numbers $(\mathrm{Re}=50-70)$ show similar results. Therefore, we choose a weaker threshold $L K^{1 / 2}>0.4$ for the $\mathrm{Y}$ and $\mathrm{Z}$ components. In addition, we apply another threshold $L>0.05$ to rule out the extremely short ones. Higher thresholds can be used to achieve a better accuracy, but the penalty is that fewer samples are available. The $\mathrm{X}$ component error

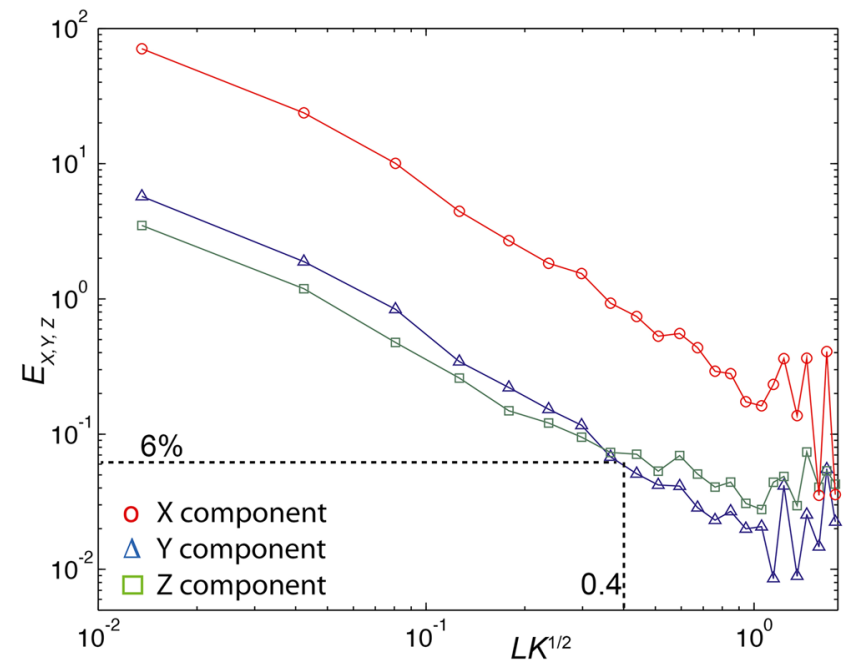

FIG. 4. The accuracy and arc length relation. The uncertainty is defined as the average difference between the measurement values and the analytical solution, normalized by the vorticity magnitude, $E=\left|\omega_{i}-\omega_{\text {Ana }, i}\right||| \omega_{A n a} \mid$. At $L K^{1 / 2}=0.4$, the uncertainty of $Y$ and $Z$ components reaches an acceptable level of $6 \%$. For $L K^{1 / 2}$ $>0.8$, the uncertainty reaches the minimum. is in the order of $50 \%$ for $L K^{1 / 2}=0.4$. To obtain an accurate $\omega_{x}$, the strong criterion $L K^{1 / 2}>0.8$ must be used and the uncertainty is in the order of $20 \%$. These results are consistent with the theoretical error estimation in Sec. II.

Since the relative error is of the same magnitude for all the low Re number cases in the stable Couette flow. We collect all the trajectory samples that satisfy our criteria and investigate the probability
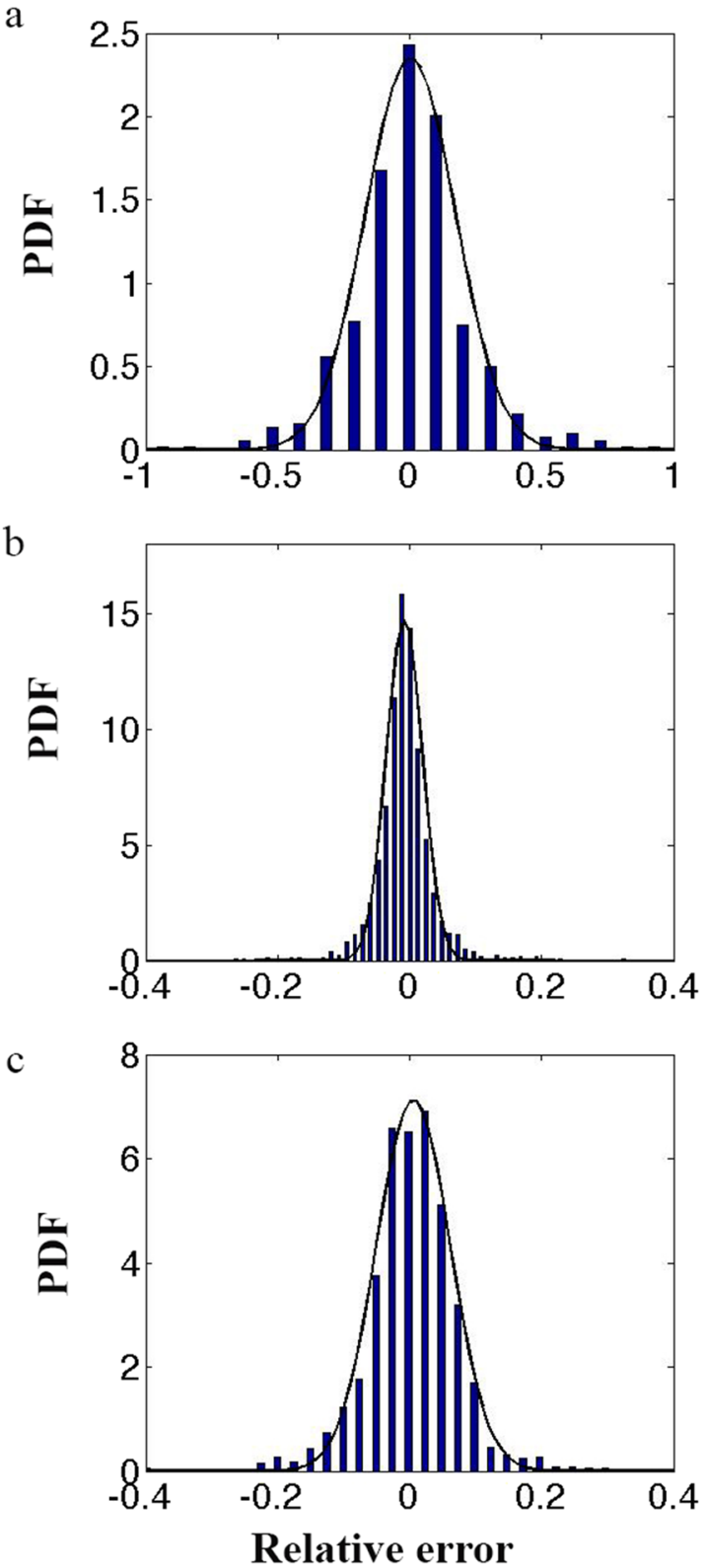

FIG. 5. The probability density distribution of uncertainties for $X, Y$, and $Z$ components [from (a) to (c)]. They all have Gaussian profiles. Obviously, the $X$ component has a much wider distribution as the error in this direction is inherently larger. The standard deviations of the Gaussian fittings are $\sigma=0.17,0.027$, and 0.056 , respectively. 
density distribution of these instantaneous samples. As shown in Fig. 5, these distributions have Gaussian profiles. The mean values of the relative errors are 0 for all the three components. By numerically fitting the profile, we obtain their standard deviations: $2.7 \%$, $5.6 \%$, and $17 \%$ for the $\mathrm{Y}, \mathrm{Z}$, and $\mathrm{X}$ components. Therefore, according to the $4 \sigma$ rule, $99.9 \%$ samples have accuracy of $\pm 5.4 \%, \pm 11.2 \%$, and $\pm 34 \%$ in $\mathrm{Y}, \mathrm{Z}$, and $\mathrm{X}$ directions, respectively.

\section{B. Flow transition in the Taylor-Couette flow}

To describe the flow field, the cylindrical coordinate $(z, r, \theta)$ is used in this subsection. The average vorticity at different $R e$ numbers is shown in Fig. 6(a). The vorticity measured in the stable Couette flow regime $R e=50-78$ agrees with the analytical solution. At slightly larger $R e$ numbers, the mean vorticity deviates from the projected analytical results as Taylor vortices begin to form. Due to the mixing caused by Taylor vortices at very high $R e$, the average axial vorticity near the outer cylinder walls is usually larger than

a

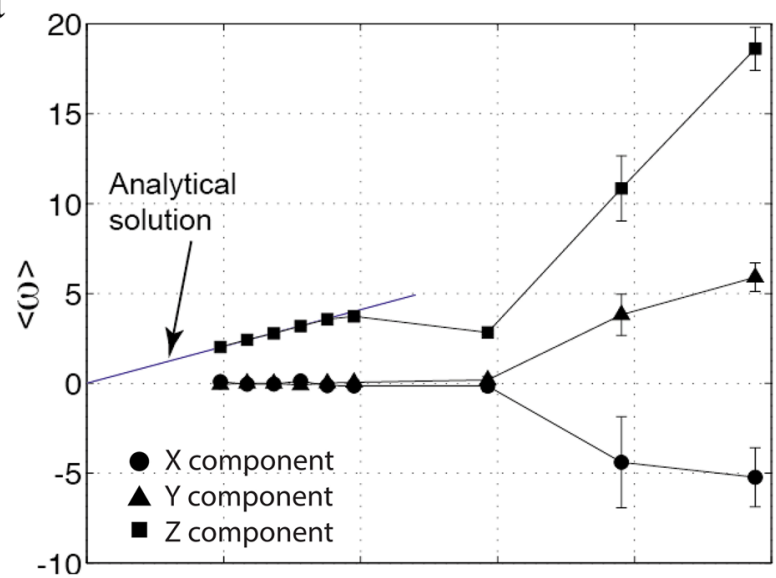

b

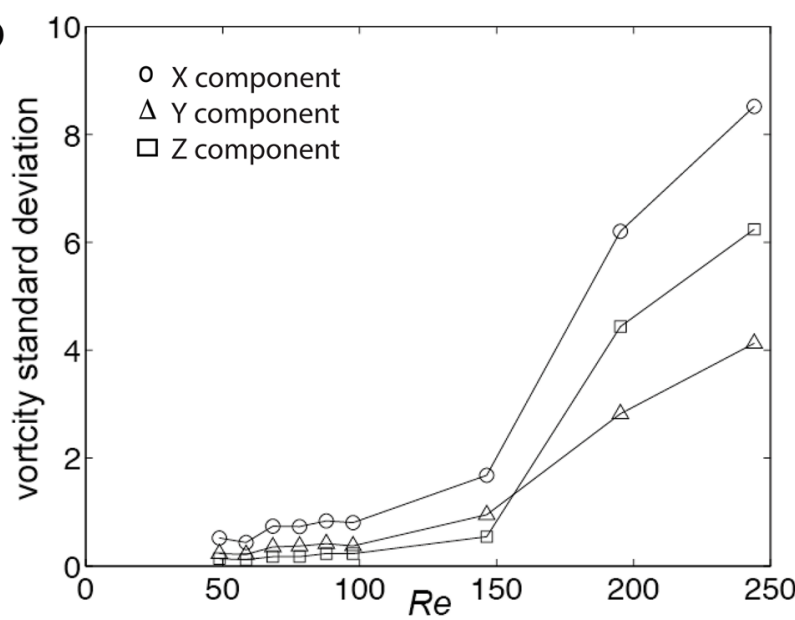

FIG. 6. The mean value (a) and standard deviation (b) of the measured vorticity at different $R e$ numbers. The consistency between the analytical solution and the mean at low $R e$ is evident. As the flow transits, the measured vorticity deviates from the analytical solution. the projected analytical solution. Our measurements, which were performed near that wall, indeed show this trend at $R e>200$. The standard deviation of vorticity is in Fig. 6(b). At low Re number, they are very small, representing the measurement uncertainty. As the Re number passes the critical value, the vorticity inside the measurement domain is not uniform any more and the current measurement method does not distinguish particles positions in the configuration space. As a result, the standard deviations increase significantly.

\section{SUMMARY AND OUTLOOK}

We have developed a vorticity measurement technique that is based on the idea proposed by Frish and $\mathrm{Webb}^{17}$ to directly measure the rotation of fluid elements using mirror-encapsulated microparticles. The spatial resolution, given by the size of the particles, can be an order of magnitude higher than that of conventional methods such as hot-wire arrays and PIV. However, the reconstruction of particle rotation has large uncertainties, which is a disadvantage, because the system is inherently insensitive to one component of the rotation. The solution is to enlarge the numerical aperture of the optical system that collects the reflections from the encapsulated mirrors. A spherical concave mirror serves this purpose better than a spherical lens. The setup of placing the screen at the focal plane of the concave mirror (or spherical lens) eliminates the effect of particle translation. The theoretical analysis shows how the relative errors depend on parameters of the experimental setting, and the results are confirmed experimentally. The errors of three vorticity components in the studied flow are approximately $\pm 5 \%$ for two vorticity components and $\pm 15 \%$ for the third one. Although we cannot obtain the same uncertainty for all components in the instantaneous measurement, it is possible to add a second optical system to achieve the same accuracy for statistical results of each component.

The performance of the new technique is demonstrated using the transition of a Taylor-Couette flow from the Couette to Taylorvortex regime. The high spatial and temporal resolutions of the technique make it an ideal tool to study vorticity dynamics in highReynolds-number turbulence, e.g., how vorticity statistics scale with the Reynolds number. ${ }^{29}$ Furthermore, the temporal evolution of vorticity along the particle trajectory in turbulence is even more interesting. ${ }^{20,30-32}$ Therefore, in the future, we will combine our current setup with the standard Lagrangian particle tracking ${ }^{33}$ to study the vorticity evolution.

\section{ACKNOWLEDGMENTS}

The authors thank Dr. W. Webb and Dr. D. Ferguson at Cornell University for suggesting the optical vorticity probe technique to us. We are also grateful to Dr. A. Kubitzek, Mr. D. Wolf, and Mr. A. Kopp at MPI for Dynamics and Self-Organization for their assistance in designing and manufacturing the apparatus and Mr. Kluge and Mr. Kleinhaus at MPI for Biophysical Chemistry, Göttingen, for the machining of special optics. We are grateful to Professor Landfester and Professor Weiss at MPI for Polymer Research, Mainz, for their help in preparing the mirror-encapsulated particles and to Mr. Mónico for the helpful discussions of Taylor-Couette flows. We acknowledge the financial support from the Max Planck Society. H.W. also thanks the Alexander von Humboldt Foundation for the financial support through a fellowship. 


\section{REFERENCES}

${ }^{1} \mathrm{~J}$. M. Wallace, "Twenty years of experimental and direct numerical simulation access to the velocity gradient tensor: What have we learned about turbulence?," Phys. Fluids 21, 021301 (2009).

${ }^{2}$ J. M. Wallace and P. V. Vukoslavčević, "Measurement of the velocity gradient tensor in turbulent flows," Annu. Rev. Fluid Mech. 42, 157-181 (2010).

${ }^{3}$ L. S. G. Kovasznay, "Physical measurements in gas dynamics," in High Speed Aerodynamics and Jet Propulsion (Princeton University Press, 1954), Vol. 9, pp. 213-285.

${ }^{4}$ G. Gulitski, M. Kholmyansky, W. Kinzelbach, B. Lüthi, A. Tsinober, and S. Yorish, "Velocity and temperature derivatives in high-Reynolds-number turbulent flows in the atmospheric surface layer. Part 1. Facilities, methods and some general results," J. Fluid Mech. 589, 57-81 (2007)

${ }^{5}$ D. B. Lang and P. Dimotakis, "Measuring vorticity using the laser Doppler velocimeter,” Bull. Am. Phys. Soc. 27, 1166 (1982).

${ }^{6}$ G. R. Romano, R. A. Antonia, and T. Zhou, "Evaluation of LDA temporal and spatial velocity structure functions in a low Reynolds number turbulent channel flow," Exp. Fluids 27, 368-377 (1999).

${ }^{7}$ J. H. Agui and Y. Andreopoulos, "A new laser vorticity probe-LAVOR: Its development and validation in a turbulent boundary layer," Exp. Fluids 34, 192-205 (2003)

${ }^{8}$ J. Westerweel, G. E. Elsinga, and R. J. Adrian, "Particle image velocimetry for complex and turbulent flows,” Annu. Rev. Fluid Mech. 45, 409-436 (2013).

${ }^{9}$ J. Katz and J. Sheng, "Applications of holography in fluid mechanics and particle dynamics,” Annu. Rev. Fluid Mech. 42, 531-555 (2010)

${ }^{10}$ G. E. Elsinga, F. Scarano, B. Wieneke, and B. W. van Oudheusden, "Tomographic particle image velocimetry,” Exp. Fluids 41, 933-947 (2006)

${ }^{11}$ B. W. Zeff, D. D. Lanterman, R. McAllister, R. Roy, E. J. Kostelich, and D. P. Lathrop, "Measuring intense rotation and dissipation in turbulent flows," Nature 421, 146-149 (2003).

${ }^{12}$ S. G. Hanson, "Application of the laser gradient anemometer for fluid flow measurements," in 2nd Symposium on Application of Laser Anemometry to Fluid Mechanics, 1984.

${ }^{13}$ M. V. Otugen, W.-J. Su, and G. Papadopoulos, “A new laser-based method for strain rate and vorticity measurements," Meas. Sci. Technol. 9, 267-274 (1998).

${ }^{14} \mathrm{P}$. Tong and W. I. Goldburg, "Relative velocity fluctuations in turbulent flows at moderate Reynolds numbers. I. Experimental,” Phys. Fluids 31, 2841-2848 (1988).

${ }^{15}$ P. Tong, W. I. Goldburg, J. S. Huang, and T. A. Witten, “Anisotropy in turbulent drag reduction,” Phys. Rev. Lett. 65, 2780-2783 (1990).

${ }^{16} \mathrm{H}$. Kriegs and W. Staude, "A laser pulse technique for the measurement of time-resolved velocity gradients in fluid flow," Meas. Sci. Technol. 6, 653-662 (1995).
${ }^{17}$ M. B. Frish and W. W. Webb, "Direct measurement of vorticity by optical probe," J. Fluid Mech. 107, 173-200 (1981).

18 A. Belmonte, C. Rosales-Guzmán, and J. P. Torres, "Measurement of flow vorticity with helical beams of light," Optica 2, 1002-1005 (2015).

${ }^{19}$ A. Ryabtsev, S. Pouya, A. Safaripour, M. Koochesfahani, and M. Dantus, "Fluid flow vorticity measurement using laser beams with orbital angular momentum," Opt. Express 24, 11762 (2016).

${ }^{20}$ R. Zimmermann, Y. Gasteuil, M. Bourgoin, R. Volk, A. Pumir, and J.-F. Pinton, "Tracking the dynamics of translation and absolute orientation of a sphere in a turbulent flow,” Rev. Sci. Instrum. 82, 033906 (2011).

${ }^{21}$ G. G. Marcus, S. Parsa, S. Kramel, R. Ni, and G. A. Voth, "Measurements of the solid-body rotation of anisotropic particles in 3D turbulence," New J. Phys. 16, 102001 (2014)

${ }^{22}$ K. A. Buist, P. Jayaprakash, J. A. Kuipers, N. G. Deen, and J. T. Padding, "Magnetic particle tracking for nonspherical particles in a cylindrical fluidized bed," AIChE J. 63, 5335-5342 (2017).

${ }^{23} \mathrm{X}$. Tao and $\mathrm{H}$. Wu, "A comparison of the sequential quadratic programing algorithm and extended Kalman filter method in the magnetic particle tracking reconstruction," in AIAA Scitech 2019 Forum (AIAA, 2019), p. 272.

${ }^{24} \mathrm{X}$. Tao, X. Tu, and $\mathrm{H}$. Wu, "A new development in magnetic particle tracking technology and its application in a sheared dense granular flow," Rev. Sci. Instrum. 90, 065116 (2019).

${ }^{25}$ W. W. Webb and R. D. Ferguson, "Multidimensional vorticity measurement optical probe system," U.S. Patent No. 4,664,513 (12 May 1987).

${ }^{26}$ G. B. Jeffery, "The motion of ellipsoidal particles immersed in a viscous fluid," Proc. R. Soc. London 102, 161-179 (1922)

${ }^{27} \mathrm{H}$. Brenner, "The Stokes resistance of an arbitrary particle-III: Shear fields," Chem. Eng. Sci. 19, 631-651 (1964).

${ }^{28}$ A. Recktenwald, M. Lucke, and H. W. Muller, "Taylor vortex formation in axial through-flow: Linear and weakly nonlinear analysis," Phys. Rev. E 48, 4444 (1993).

${ }^{29} \mathrm{H}$. Xu, N. T. Ouellette, and E. Bodenschatz, "Curvature of Lagrangian trajectories in turbulence,” Phys. Rev. Lett. 98, 050201 (2007).

${ }^{30} \mathrm{H}$. Xu, A. Pumir, and E. Bodenschatz, "The pirouette effect in turbulent flows," Nat. Phys. 7, 709-712 (2011).

${ }^{31}$ S. Parsa, E. Calzavarini, F. Toschi, and G. A. Voth, "Rotation rate of rods in turbulent fluid flow,” Phys. Rev. Lett. 109, 134501 (2012).

${ }^{32}$ A. Pumir, E. Bodenschatz, and H. Xu, "Tetrahedron deformation and alignment of perceived vorticity and strain in a turbulent flow," Phys. Fluids 25, 03510 (2013).

${ }^{33}$ N. T. Ouellette, H. Xu, and E. Bodenschatz, "A quantitative study of threedimensional Lagrangian particle tracking algorithms,” Exp. Fluids 40, 301-313 (2006). 